\title{
Achieving QoS in Virtual MIMO systems: a satisfaction equilibrium analysis
}

\author{
Hassan Bennani ${ }^{1}$, Essaid Sabir ${ }^{2 *}$, Abdellatif Kobbane ${ }^{1}$, Jalel Ben-Othman ${ }^{3}$ and Mohamed El-koutbi ${ }^{1}$
}

\begin{abstract}
This paper presents a game theoretic framework to analyze a cellular-WLAN heterogeneous network from a virtual multiple-input multiple-output (VMIMO) perspective. Namely, we restrict to the uplink case and consider a non-cooperative game where each user seeks to meet some quality of service (QoS). Moreover, mobile users are allowed to re-inject, through their WLAN interfaces, a part of their throughput in the induced game. Thus, the interaction among users defines a distributed VMIMO with a possibly throughput exchange. This mechanism will help all users to meet their respective QoS expressed by the perceived average throughput. The average throughput is considered to be the utility function used in this framework. Now, each mobile user experiences a certain throughput composed of two parts: (1) the throughput received from cellular subsystem and (2) the throughput received from WLAN subsystem. Naturally, we use the concept of satisfaction equilibrium to predict the behavior of the network. Indeed, we provided a sufficient condition for the existence of such an equilibrium. Next, we prove the uniqueness of the equilibrium and compute it explicitly. Afterward, we propose a fully distributed algorithm inspired from the well-known Banach-Picard learning algorithm. Our scheme has many good features facilitating its implementation and usability. Indeed, it accurately converges to the equilibrium (if exists), it is very fast, and it requires no external information. Simulation results validate the algorithm and show its robustness and illustrate numerically the proposed learning scheme for quality-of-service management in such a heterogeneous network.
\end{abstract}

Keywords: Virtual multiple input multiple output, Quality of service, Game theory, Satisfaction equilibrium, Cellular/WLAN heterogeneous network

\section{Introduction}

Nowadays, users increasingly need higher throughput for better use of applications and new services such as streaming videos, files transfer, and 3D gaming. Through time, many techniques have been developed to satisfy that need. Among the techniques developed are frequency division multiple access (FDMA) and time division multiple access (TDMA) in the $2 \mathrm{G}$, code division multiple access (CDMA) in $3 \mathrm{G}$, and orthogonal frequency division multiple access (OFDMA) and multiple-input multiple-output (MIMO) in $4 \mathrm{G}$.

Figure 1 shows simple versions of various antenna techniques. The name of each technique defines how the radio channel is accessed by the transmitters and receivers. The single-input single-output (SISO) approach is the basic

*Correspondence: e.sabir@ensem.ac.ma

${ }^{2}$ NEST Research Group, ENSEM, Hassan II University, Route d'El Jadida BP 8118 Oasis, 20000 Casablanca, Morocco

Full list of author information is available at the end of the article radio-channel access mode with one transmitter and one receiver. The multiple-input single-output (MISO) mode is more complex, using two or more transmit antennas and a single receiver antenna. In this mode, which is commonly called a transmit diversity system, the same data is sent to both of the transmitting antennas but is coded in such a way that the receiver can identify each transmitter. The single-input multiple-output (SIMO) approach, often referred to as a receive diversity technique, utilizes one transmit antenna and two or more receiver antennas.

MIMO technology requires two or more antennas at transmission and two or more antennas at reception. This mode is not just a combination between MISO and SIMO because multiple data streams are transmitted simultaneously in the same frequency and time, taking full advantage of the different paths in the radio channel. MIMO have been amply recognized as promising techniques in order to enhance and manage effectively the spectral and resources mobile communication systems. Several 


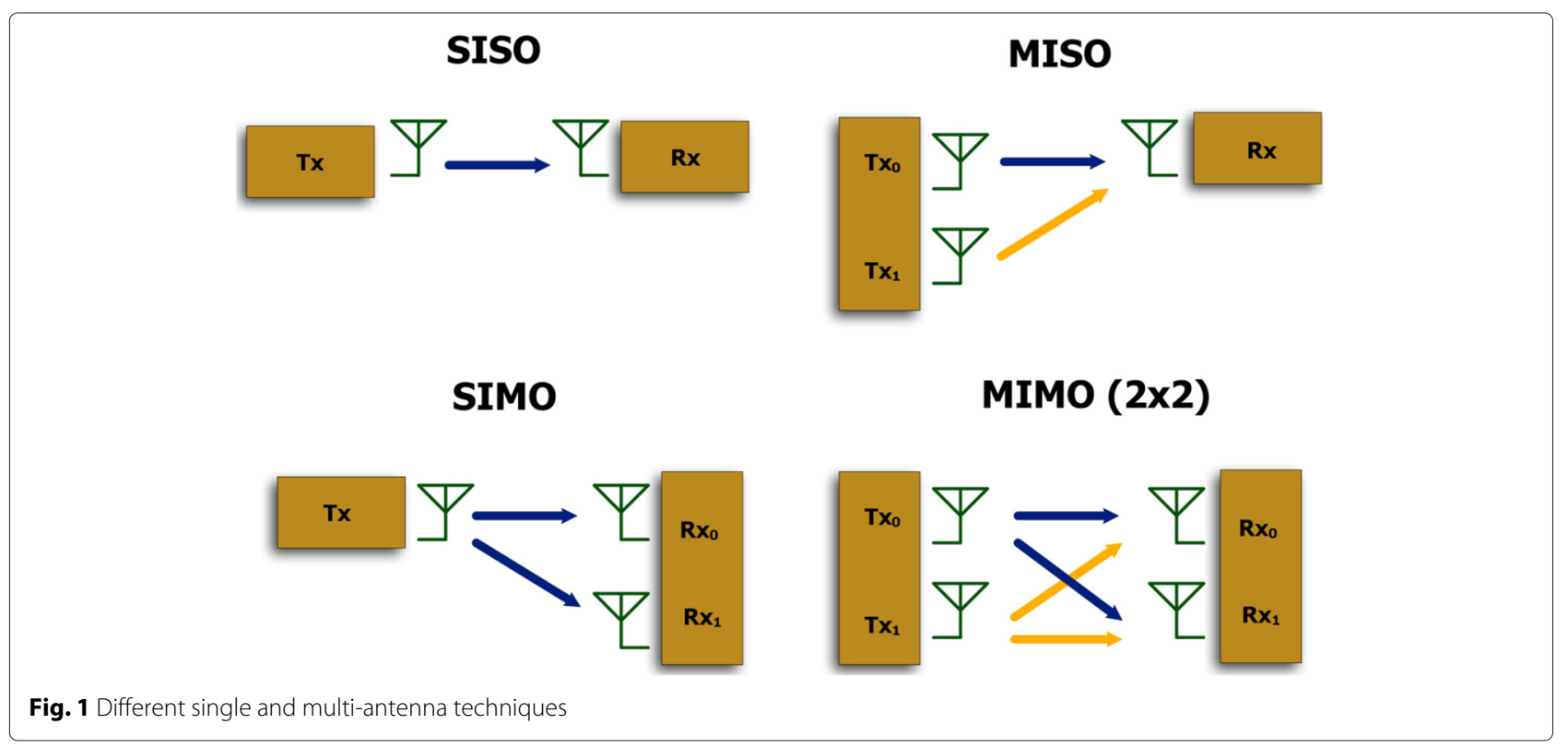

international standards such as LTE-A (LTE-advanced), 3GPP LTE (third-generation partnership project longterm evolution), and IEEE $802.16 \mathrm{e} / \mathrm{m}$ have supported the MIMO techniques. However, some challenges such as implementing a conventional antenna seem a problematic challenge due to very small size mobile transmitter/receiver. The spatial diversity can be realized by cooperation between mobile terminals, to form a relaying system through a multiple single antenna mobile. This mechanism is called a virtual MIMO system (VMIMO). In this system, the terminal antennas form a virtual antenna array with a virtual joint. Nowadays, the research area around VMIMO know a significant grow for the purpose to study and understand it. For the fourth-generation and fifth-generation mobile communications technologies, VMIMO is considered one of the most crucial technologies enabling new paradigms such Internet of Things and device-to-device communications.

The benefits of MIMO technologies are the damping the cost of multiple antennas by the use of all neighboring terminals, which gives us promising opportunities to increase the capacity of the system and to enhance error rate performance. However, the same fundamental dams need to be analyzed in order to take full advantage of VMIMO. First, in cellular network, only a limited number of terminals can be supported to form a VMIMO system. This restriction is due to the limited processing capability of base stations. Hence, the choice of mobile terminals to form a gainful VMIMO becomes a huge challenge. Secondly, in an efficient VIMIMO system, the information exchanged between mobile terminals must be minimized as much as possible. Furthermore, at the base stations, the channel state information of each mobile terminal is required to carry out pre-coding in order to drive the signaling load, particularly in the frequency division duplexing (FDD) mode. Figure 2 illustrates a global VMIMO communications scheme for network users among multiple technologies. Thus, it is very important to study the interaction and the impact of the overhead on the global performance of the system. Mobile terminals must be equipped with an innovative mechanism in order to return sufficient information with less signaling. On one hand, it is necessary to study the impact of the overhead on the overall performance of the system. On the other hand, innovative schemes are required for mobile terminals to feed back sufficient information via limited signaling. Therefore, collaboration among mobile terminals could be implemented in future cellular systems, and the cost of using the collaboration between terminals must be evaluated in VMIMO.

The rest of this manuscript is organized as follows: Section 2 discusses and presents a detailed review on related literature. Section 3 presents a simple noncooperative game for the VMIMO system with possible throughput re-injection. Next, we provide a complete analysis of the induced game and characterize the equilibria points (existence, uniqueness, and computation) in Section 4. A fully distributed algorithm to discover the satisfaction equilibrium is presented in Section 5. Extensive numerical examples and simulations are given in Section 6. We finally draw a conclusion and give some future directions in Section 7.

\section{Related work}

Many studies have been published which used multiple approaches using the power control algorithms and game 


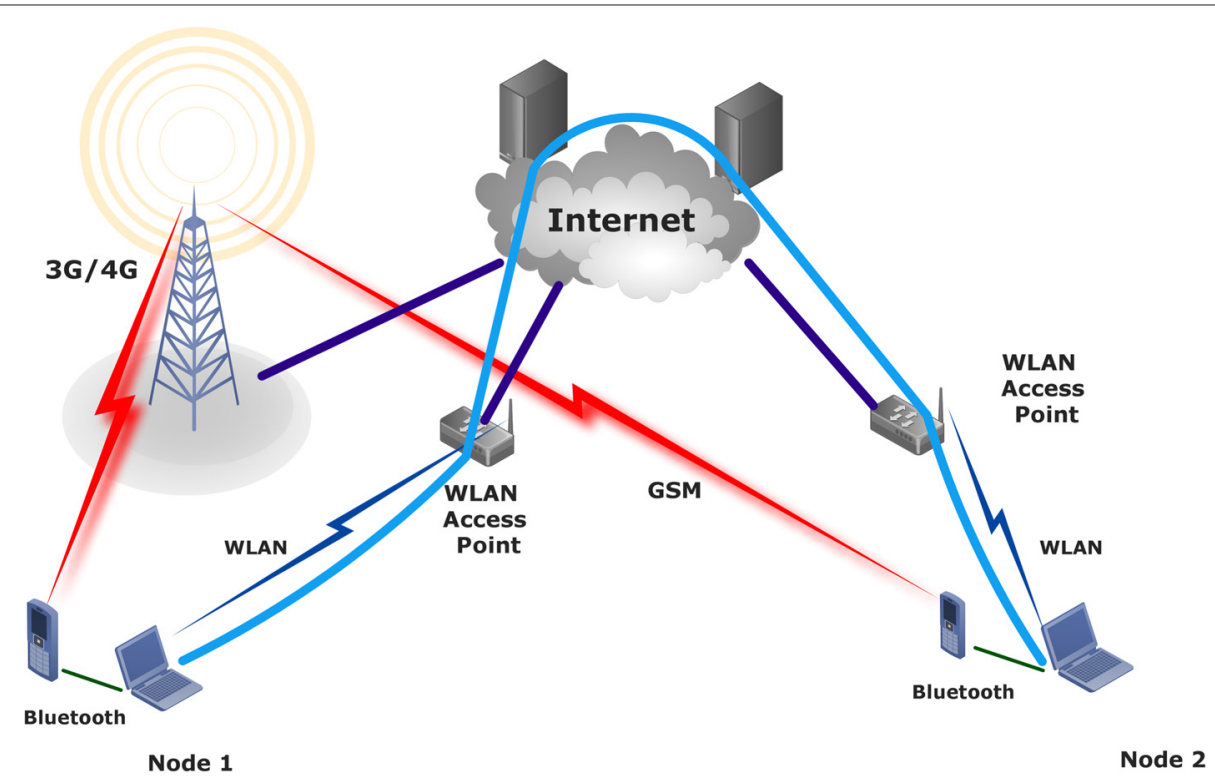

Fig. 2 Virtual MIMO system model

theory. In [1], the authors present the power control in cellular radio systems from an economic point of view and study the uplink power control problem as a noncooperative N-person game. Goodman et al. [2] implement a new algorithm network assisted power control, in order to study the impact of effective power control on the system quality and efficiency in wireless communications. The authors in [3], study the effect of pricing transmit power in a multi-cell wireless data system, and introduce pricing of transmit power as a mechanism for influencing data user behavior. One of the challenges of wireless communications systems is the management and the efficient use of radio resources. In [4], the authors present a power control solution for wireless data in the analytical setting of a game theoretic framework and introduce pricing of transmit powers in order to obtain Pareto improvement of the non-cooperative power control game.

T. Alpcan et al. [5] present a new game theoretic treatment of distributed power control in CDMA wireless systems in order to study power control problem, pricing and allocation of a single resource among several users. In [6], a game theoretic approach in multirate code-division multiple access system, in order to maximize the total system throughput by means of power control is presented. Objectives of most radio resource-management schemes can be classified as either user-centric or network-centric. Authors in [7] consider the joint optimization of both user-centric and network-centric metrics. They use a utility function as the user-centric metric and introduce an explicit pricing mechanism to mediate between the user-centric and network-centric resource-management problems. F. Meshkati et al. propose in [8] a power control game for MC-CDMA systems; it is shown that the proposed approach results in a significant improvement in the total utility achieved at equilibrium. Cui et al. [9] presents an analysis of the best modulation and transmission strategy to minimize the total energy consumption in a radio application in sensor networks. By considering different MIMO systems' schemes, it is shown that tremendous energy saving is possible for transmission distances larger than a given threshold, and over some distance, cooperative MIMO can achieve both energy and delay.

S. K. Jayaweera et al. [10] proposed a VMIMO scheme for distributed and cooperative wireless sensor networks, where the energy and delay efficiencies are derived using semi-analytic techniques. The author shows in this study that with judicious choice of design parameters the VMIMO technique can be made to provide significant energy and delay efficiencies. In [11], a new multi-hop VMIMO communication protocol is proposed by the cross-layer design to jointly improve the energy efficiency, reliability, and end-to-end (ETE) QoS provisioning in wireless sensor network (WSN). Simulation results of this work show the effectiveness of the proposed protocol in energy saving and QoS provisioning. Traditional VMIMO transmission schemes principally focus on maximizing the throughput of grouped mobile terminals without taking into account the quality-of-service (QoS) provisioning. The authors in [12] propose the optimal power allocation schemes with statistical QoS provisioning to maximize the effective capacity of noncollaborative/collaborative VMIMO wireless networks, 
respectively. Xu Hongli et al. study in [13] the problem of constructing an energy-efficient topology in wireless sensor networks using VMIMO communication. The simulation results show that the VMIMO topology control helps to decrease the power consumptions by approximately $32 \%$ compared to the existing algorithms.

In [14], the author examine a tax inspired mechanism design to Achieve QoS in VMIMO Systems using a game theoretic approach. The utility function used in the study is defined so that the throughput used by the user is divided into two components: the throughput received from cellular Network, and throughput received from Virtual MIMO System. The study has shown that there is a unique constrained Nash equilibrium for the proposed game when the sum of demanded QoS for all users is bounded.

MIMO and smart antenna techniques have been extensively used as promising schemes to improve the spectrum efficiency. The authors in [15] propose a VMIMO scheme for direction of arrival estimation in which a set of user equipments are grouped together to simultaneously communicate with the base station on a given resource block and propose an automatic weighted subspace fitting algorithm that can detect the number of independent signals automatically and show accurate direction of arrival estimation. VMIMO systems present several challenges in order to improve the spatial diversity gain and spectrum efficiency. Karimi et al. [16] presents a novel solution that decomposes the VMIMO user grouping. The results of this solution under different network configuration demonstrate that it achieves much higher data throughput as compared to existing solutions.

Massive MIMO technology has received great attention recently. The main benefit of this technology can be realized only when the quality of estimated channel is ensured at both transmitter and receiver, by using a large number of antennas into the wireless transceiver. Sunho Park et al. [17] present an alternative channel estimation technique dealing with the pilot shortage in the massive MIMO systems. It is shown that the proposed method achieves substantial performance gain over conventional approaches employing pilot signals exclusively. The authors in [18] study energy-efficient data gathering in wireless sensor networks using VMIMO. The theoretical analysis and simulations show that the energy consumption decreases by 81 and $36 \%$ compared to existing solutions. Wei Lu et al. [19] propose a compressed sensing feedback scheme for zero-forcing beam-forming in order to enhance the throughput in MIMO broadcast channel. Simulation-based results show that the proposed scheme has good performances compared to existing feedback schemes. In systems based on network MIMO, the interference is one of the major performance limiting factors to form a multi-cell virtual MIMO system. The authors in
[20] develop a scalable interference coordination strategy of combining clustered network MIMO with fractional frequency reuse. It is shown that the cell rate performance of this new approach is better than that of the two existing strategies. The authors in [21] investigate the problem of distributed channel selection using a game-theoretic stochastic learning solution in an opportunistic spectrum access and propose a genie-aided algorithm to achieve the NE points under the assumption of perfect environment knowledge. The study shows that the SLA based learning algorithm achieves high system throughput with good fairness. In [22], the authors examine the problem of achieving global optimization for distributed channel selections in cognitive radio networks (CRNs), using game theoretic approach. The authors propose two special cases of local interaction game to study this problem. The results of this study shown that with the proposed games, global optimization is achieved with local information. In [23], the authors propose a scheme for channel access self-regulation. The main idea is to introduce hierarchy among mobile users. This scheme seems to behave well and succeeds to increase the bandwidth utilization, which may increase the number of mobile users to serve and therefore to enlarge the stability region.

In this paper, we consider an integrated cellular-WLAN network and we introduce an incentive mechanism for throughput recycling through WLAN interfaces. The main idea is getting inspired from the society tax system in a "give to receive fashion." Yet, each mobile user will get some given throughput from the cellular network (this can be assimilated to a salary). Next, each mobile user should re-inject a part of its throughput into the VMIMO subsystem through WLAN link (this can be assimilated to a tax to be paid to the society). Finally, each mobile user will get some extra throughput from VMIMO subsystem (this can be assimilated to facilities that society provides to everybody according to their participations). This mechanism may allow all mobile users to meet their QoS even their cellular radio condition are bad. The main contributions of this paper are as follows:

- We design a new tax-inspired mechanism appealing for cooperation in heterogeneous networks.

- We build a simple game theoretic framework to analyze the behavior of mobile users in VMIMO systems.

- To the best of our knowledge, this paper is one of the first work to deal with autonomic QoS provisioning in VMIMO systems.

- Our framework is quite simple but is adapted for device-to-device, machine-to-machine and autonomic communications; yet, both links device/base-station and device/device are modelled in a simple way. 
- A full characterization (existence, uniqueness, computation, and convergence) of the satisfaction equilibrium has been provided.

- We proposed a fully distributed (no external information is required) algorithm to discover the satisfaction algorithm.

\section{Problem formulation}

Consider a wide geographical area served by a cellular network (3G, 4G, or 5G). We assume that mobile users are randomly distributed on a plane following a Poisson point process (PPP) with density $\lambda$. Each terminal is equipped by at least two separated network interfaces ${ }^{1}$ (e.g., LTE and WLAN). This allows simultaneous or alternative connections to the two technologies according to the coverage criterion and offered services as well. Now, each mobile user could transmit/receive data over the two interfaces. Thereby, the average throughput of user $i$ could be divided into two parts: the throughput allocated by the base station and the perceived throughput from other users over the WLAN radio. A key assumption of our analysis is that each user should cooperate with the others users. This induces a behavioral issue of cooperation among users. Indeed, if nodes refuse to cooperate, then our VMIMO-based scheme is just impossible to implement. The research community is invited to gather their input in order to design efficient incentive mechanisms for cooperation. A simple incentive mechanism is to simply say that if I cooperate today with others, I should almost surely get help from others tomorrow.

\subsection{Throughput received from cellular network}

Cellular MAC layer is designed to support multimedia services (streaming, MPEG video, etc.) and very high peak bit rates. It also fully manages the bandwidth utilization for both uplink and downlink schemes by the means of the polling process. Moreover, it supports scalable QoS depending on the ongoing service by handling some parameters such as the tolerable rate, scheduling type, and delay. In this work, the base station (3G, 4G, or 5G) is assumed to handle a multiple access (such as CDMA, OFDMA, or any other multiple access technique) scheme to communicate with covered users. Now, it allocates dynamically and exclusively to each node $i$, a set of resources guaranteeing a certain throughput. We use the throughput formula defined in [8], as the net number of information bits that are transmitted without error per unit time. It can be expressed as

$$
T_{i}^{C}=\frac{L}{M} R_{i} f\left(\gamma_{i}\right)
$$

where $L$ and $M$ denote, respectively, the number of information bits and the total number of bits per packet. $R_{i}$ is the transmission rate and $\gamma_{i}$ denotes the signal to interference ratio (SIR) $)^{2}$ of user $i$. Whereas $f(\cdot)$ stands for the efficiency function representing the packet success rate (PSR), i.e., the probability that a packet is received without an error. We suppose that if a packet has one or more bit errors, it will be retransmitted entirely. The efficiency function, $f\left(\gamma_{i}\right)$, is assumed to be increasing and S-shaped (sigmoidal) with $f(\infty)=1$. We also require that $f(0)=0$ to ensure that $T_{i}^{C}=0$ when $\gamma_{i}=0$.

\subsection{Throughput received from VMIMO (through WLAN)}

In a WLAN, an ongoing transmission is successful if and only if no other transmissions overlap in time with the ongoing transmission. This claim is also true for multihop wireless networks. At any given time slot, a transmission is successful if there was at most one transmission in the interference range of the receiver. Without loss of generality, we consider that the interference range and the transmission range are the same. If any node within the transmission range $r_{t x}$ of an intended receiver transmits, it collides with the tagged transmission. Each mobile user transmits in a randomly chosen slot, over WLAN system, with the attempt probability $q_{i}$. The probability of success in order to transmit over the virtual resource (WLAN)

$$
\rho_{i}=q_{i} \prod_{j \neq i}\left(1-q_{j}\right)
$$

When a tagged user intends to transmit over WLAN system, it faces contention of its neighbors, i.e., users inside its transmission rage $r_{t x}$. The PPP is assumed to be homogenous. Thus, the average number of users inside a ring of radius $r_{t x}$ is

$$
\bar{n}=\int_{0}^{2 \pi} \int_{0}^{r_{t x}} \lambda \cdot r \cdot \alpha \cdot d r \cdot d \alpha=\lambda \pi r_{t x}^{2} .
$$

It follows that the average number of neighbors of each mobile user is $\bar{n}-1$. Without loss of generality and only for simplicity purpose, it is plausible to consider that $q_{i}=$ $q_{j} \equiv q$ (by fairness of random access methods such as IEEE 802.11 and slotted aloha). Thereby, the normalized throughput (success rate) on the WLAN interface can be expressed as

$$
\rho_{i} \equiv \rho=q(1-q)^{\lambda \pi r_{t x}^{2}-1}
$$

If we assume that the bit-rate over the WLAN interface is denoted $\eta$. Then, the effective throughput (goodput) that user $i$ experience over WLAN is written

$$
T_{i}^{W} \equiv \rho_{i} \eta
$$

\subsection{Average throughput}

In our VMIMO scheme, we consider that each user may experience a different allocated throughput by the base station. These differences are due to the running service, geographic position of mobile users, channel conditions, 
transmit power, and many other reasons. We assume along this paper that each user runs a service with some QoS requirements. For simplicity, let us consider that the QoS metric is the throughput demand that should be met in order for the service to have an acceptable quality of service and an acceptable quality of experience as well. Obviously, our study holds for other QoS metrics such as delay or jitter.

When negotiating transmission parameters needed to achieve a good service, the base station will compute the transmission power and the resource scheduling. Let $d_{i}$ be the throughput demand required by user $i$, and let D be the vector of all throughput demands. After being accepted in the network, user $i$ would be informed by appropriate parameters' values to use, e.g., the transmit power for uplink. In reality, it is almost impossible to achieve exactly the required throughput $d_{i}$. Thus, user $i$ would receive average throughput $d_{i} \pm \Delta d_{i}$. Thereby, the received throughput would be either less than or greater than what we asked for. The main two ideas of this work are (1) to give the throughput surplus to other users that need a bit and (2) to ask others to give us throughput when needed.

Let us denote by $x_{i}$ the proportion of cellular throughput that user $i$ keeps for himself/herself. The remaining throughput, i.e., $1-x_{i}$ will be re-injected in to the system and will be shared among the users inside user $i$ 's WLAN transmission range. We will refer to this quantity as the virtual throughput offered by user $i$ to its neighbors. Thus, the average throughput received by mobile user $i$ can be expressed as:

$$
\Theta_{i} \triangleq x_{i} T_{i}^{C}+\sum_{j \neq i}\left(1-x_{j}\right) T_{j}^{W} \pi_{j, i}(\mathbf{D})
$$

where $\left(1-x_{j}\right) T_{j}^{C}$ is the proportion of cellular throughput allocated by user $j$ to its neighboring users. But, the effective throughput re-injected by user $j$ in the system is only equal to $\left(1-x_{j}\right) T_{j}^{W}=\left(1-x_{j}\right) \rho_{j} T_{j}^{C}$ (collisions effect). The function $\pi_{j, i}(\cdot)$ defines how much virtual throughput offered by user $j$ should be allocated to its neighboring node $i$. To fine-tune the virtual throughput allocation, we shall assume that the sharing distribution depends on the whole demand vector $\mathbf{D}=\left(d_{1}, d_{2}, \cdots, d_{m}\right)$. Other criteria could apply as well to the sharing function.

\section{VMIMO as a non-cooperative game}

\subsection{The non-cooperative game}

We consider the simple form of the interaction among mobile users, where they only can choose the amount of throughput to keep (i.e., $x_{i}$ ) and thereafter the amount of throughput to inject in the system (i.e., $1-x_{i}$ ). Power control is assumed to be run by the base station. Each mobile user will then face the problem of deciding the appropriate amount of throughput to allocate to others. We propose a non-cooperative game where each user (selfishly) decides the amount of throughput to yield to others in order to satisfy its throughput requirement $d_{i}$.

We denote the VMIMO game on its normal form as $\mathcal{G}=[\Omega, \mathcal{A}, \Theta, \mathbf{D}]$, where $\Omega=\{1, . ., m\}$ is the set of players (mobile users), $\mathcal{A}=[0,1]^{m}$ is the space of strategies set for all users, and $\Theta=\left[\Theta_{1}, \Theta_{2}, \cdots, \Theta_{m}\right]$ is the vector of payoff functions. In our setting, the average throughput will be considered as the utility function. $\mathbf{D}=\left(d_{1}, d_{2}, \cdots, d_{m}\right)$ denotes the throughput demand vector which defines the set of objectives (QoS) to meet by mobile users.

Each mobile user is looking to experience an average throughput greater than or equal to its throughput demand required by its running services; otherwise, the service would not discard. Then, the interaction among mobile users can be clearly explained as follows:

Step 1 : Mobile user $i$ receives an average throughput $T_{i}^{C}$ from the cellular networks. This received throughput may be greater than, less than, or equal to its required throughput demand $d_{i}$.

Step 2 : Mobile user $i$ decides the amount of throughput $x_{i}$ to keep for itself. Then $1-x_{i}$ represents the amount of throughput to re-inject into the system through WLAN interface. We refer to this as a virtual throughput since it will be shared according to some sharing rule by the other mobile users.

Step 3 : Each mobile user will fine-tune unilaterally the amount of throughput to keep (alternatively to re-inject into the WLAN) until its average total throughput is equal to its throughput demand.

\subsection{Feasible strategy and satisfactory solution}

When one imposes constraints over the payoff functions that each mobile user obtains or over the action that a player can choose in the game $\mathcal{G}$, it becomes plausible to replace the Nash equilibrium concept by a constrained (Nash) equilibrium. Here, in the presence of QoS constraints, the set of individual actions reduces to the set of actions which verifies the constraints given the actions adopted by the other mobile users.

Definition 1. Let $\mathbf{X}_{-i}=\left(x_{1}, x_{2}, \ldots, x_{i-1}, x_{i+1}, \ldots, x_{m}\right)$ be the strategy vector of all users but the ith user. a strategy $x_{i}^{*}$ of mobile user $i$ is said to be a feasible strategy iff

$$
\Theta_{i}\left(x_{i}^{*}, \mathbf{X}_{-i}\right) \geq d_{i}
$$

From now on, each mobile user will seek to achieve its throughput demand by fine-tuning the amount of throughput to re-inject in the WLAN subsystem. 
Definition 2. A strategy profile $\mathbf{X}^{*}=\left(x_{1}^{*}, x_{2}^{*}, \ldots, x_{m}^{*}\right)$ is a satisfaction Nash equilibrium point (SNEP) iff

(a) All individual throughput demand are met. Namely,

$$
\Theta_{i}\left(\mathbf{X}^{*}\right) \geq d_{i}, \quad \forall i
$$

and

(b) $\mathbf{X}^{*}$ is a feasible strategy profile.

In the remainder, we will be interested in the case of strict equality. This is not a restriction but a plausible point that has sense if the required demand is equal to the rate at which packets are generated. Moreover, energy efficiency considerations can also be a good reason to only address the case of strict equality.

\subsection{Existence and uniqueness of a SNEP}

We turn now to prove the existence/uniqueness of the SNEP point and to characterize it for the one-shot version of $\mathcal{G}$. When a SNEP (if exists) $\mathbf{X}^{*}=\left(x_{1}^{*}, x_{2}^{*}, \ldots, x_{m}^{*}\right)$ is achieved, each mobile user should met its own throughput demand, see Definition 2. Namely, we have

$$
x_{i}^{*} T_{i}^{C}+\sum_{j \neq i}\left(1-x_{j}^{*}\right) T_{j}^{W} \pi_{j, i}(\mathbf{D})=d_{i}, \quad \forall i
$$

Let $\mathbf{X}=\left(x_{1}, x_{2}, \cdots, x_{m}\right)$ be the column vector of the unknowns. Thus, we can write the non-cooperative VMIMO problem as the following non-homogeneous linear system:

$$
\mathbf{A} \cdot \mathbf{X}=\underline{\mathbf{D}},
$$

where $\mathbf{A}$ is a square matrix of size $\# m$, and which is given explicitly by the following
Table 1 Simulation parameters

\begin{tabular}{ll}
\hline Parameter & Value \\
\hline$p_{i}$ (power of user i) & $7 \mathrm{~W}$ \\
$\sigma^{2}$ (Gaussian noise) & $25 \times 10^{-16} \mathrm{~W}$ \\
$\mathrm{R}$ & $100 \mathrm{Kbps}$ \\
$\mathrm{M}$ & 100 \\
$\mathrm{~L}$ & 100 \\
\hline
\end{tabular}

$\mathbf{A}=\left(\begin{array}{cccc}T_{1}^{C} & -T_{2}^{W} \pi_{1,2}(\mathbf{D}) & \ldots & -T_{m}^{W} \pi_{1, m}(\mathbf{D}) \\ -T_{1}^{W} \pi_{2,1}(\mathbf{D}) & T_{2}^{C} & \ldots & -T_{m}^{W} \pi_{2, m}(\mathbf{D}) \\ \vdots & \vdots & \vdots & \vdots \\ -T_{1}^{W} \pi_{m, 1}(\mathbf{D}) & -T_{2}^{W} \pi_{m, 2}(\mathbf{D}) & \ldots & T_{m}^{C}\end{array}\right)$

and

$$
\underline{\mathbf{D}}=\left(\begin{array}{c}
d_{1}-\sum_{j \neq 1} T_{j}^{W} \pi_{1}(\mathbf{D}) \\
d_{2}-\sum_{j \neq 2} T_{j}^{W} \pi_{2}(\mathbf{D}) \\
\vdots \\
d_{K}-\sum_{j \neq K} T_{j}^{W} \pi_{m}(\mathbf{D})
\end{array}\right)
$$

Lemma 1. If $\mathbf{A}$ is a Cramer invertible matrix and $\mathbf{A}^{-1}$. $\underline{\mathbf{D}} \in[0,1]^{m}$. Then, the game $\mathcal{G}$ has a unique pure satisfaction equilibrium given by $\mathbf{X}^{*}=\mathbf{A}^{-1} \underline{\mathbf{D}}$.

Proof. It is straightforward that the column and the row vectors of the matrix $A$ are non-collinear, therefore it is a Cramer matrix with non-zero determinant. Henceforth, the system has a unique solution given by $\mathbf{X}^{*}=\mathbf{A}^{-1} \underline{\mathbf{D}}$.

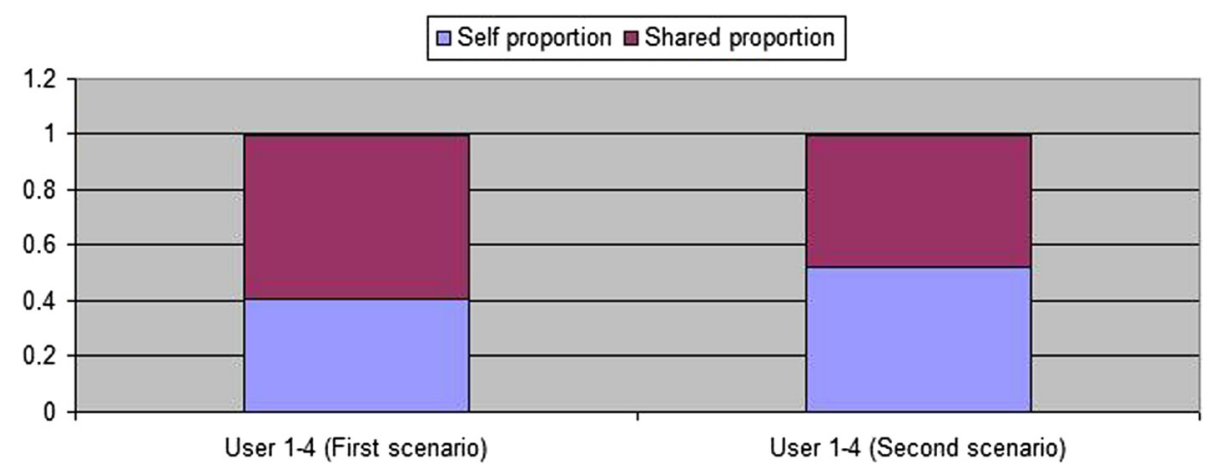

Fig. 3 Distribution of self and shared uplink proportion for four users for both scenarios 


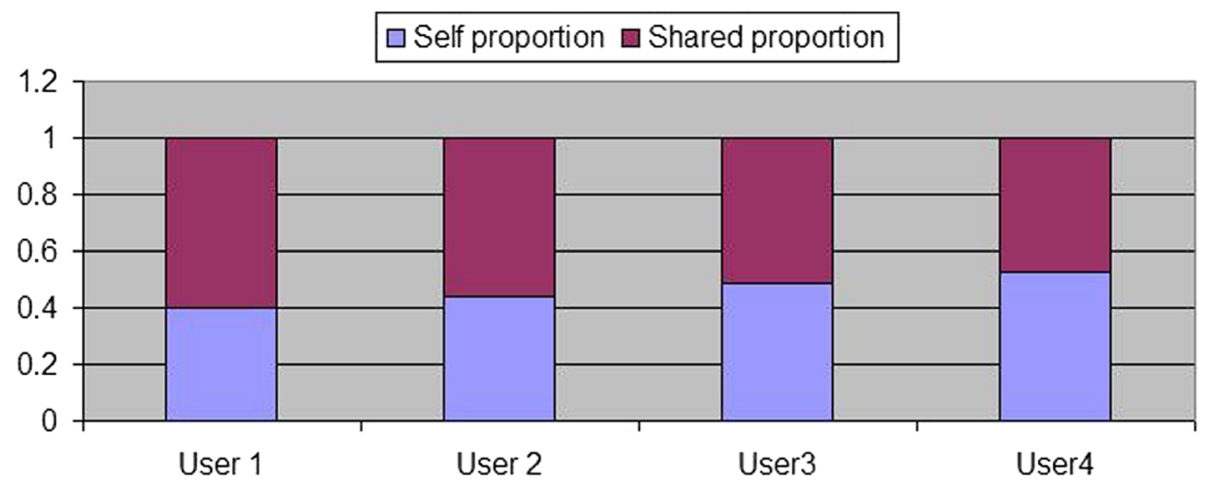

Fig. 4 Distribution of self and shared uplink proportion for four users in first scenario

\subsection{A sufficient condition for existence of a symmetric SNEP}

Now, we seek to derive a sufficient condition for existence of a satisfaction equilibrium. For that, we imagine an equivalent network with symmetric setting, with same radio conditions and with same parameters for all mobile users. This symmetric network has the same number of users and will only be used to derive an upper bound on the total demand such that an satisfaction equilibrium still exists. We set for the equivalent network $R_{i}=$ $R_{j}=R, L_{i}=L_{j}=L, d_{i}=d_{j}=d, \rho_{i}=\rho_{j}=\rho$, $f\left(\gamma_{i}\right)=f\left(\gamma_{j}\right)=f(\gamma)$, and $\pi_{i, j}(\cdot)=\pi_{j, i}(\cdot)=\pi(\cdot) \forall i, j$. Here, one consider the worst case where all mobile users ask for a throughput demand $d=\max _{i} d_{i}$. Note that the total demand generated by the symmetric network is $m \cdot d$.

The idea behind derivation of our sufficient condition is the following simple reasoning: A unique satisfaction equilibrium will exist if and only if the total throughput demand $\sum_{i=1}^{i=m} d_{i}$ does not exceed the achievable throughput (total capacity) of the network. We have the following result:

Proposition 1. Existence of a satisfaction equilibrium is guaranteed if the total throughput demand $\sum_{i=1}^{i=m} d_{i}$ verifies

$$
\sum_{i=1}^{i=m} d_{i} \leq m d \leq \frac{m L R}{M} f(\gamma) \cdot \min ((m-1) \rho \pi(d), 1) .
$$

Proof. Let $\Xi \triangleq \sum_{i=1}^{i=m} \Theta_{i}$ be the total average throughput experienced by mobile users altogether. From Eq. (6), we have:

$$
\Xi=\sum_{i=1}^{i=m}\left[x_{i} \frac{L_{i}}{M_{i}} R_{i} f\left(\gamma_{i}\right)+\sum_{j \neq i}\left(1-x_{i}\right) \rho_{j} \frac{L_{j}}{M_{j}} R_{j} f\left(\gamma_{j}\right) \pi_{j, i}(\mathbf{D})\right]
$$

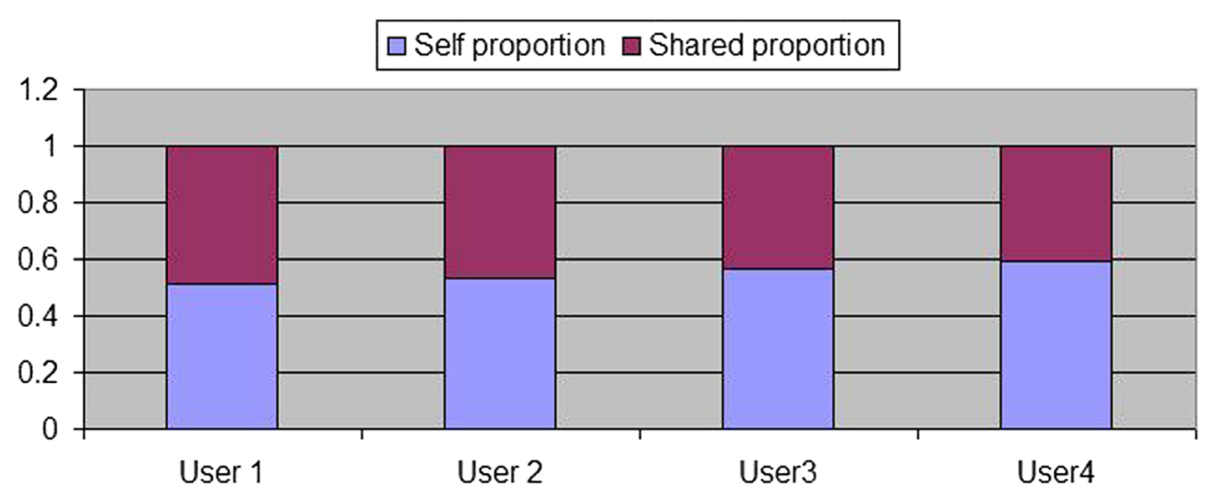

Fig. 5 Distribution of self and shared uplink proportion for four users in second scenario 
After some algebra, the total experienced throughput for the symmetric equivalent network writes:

$$
\begin{aligned}
\Xi_{\text {sym }}= & \frac{m L R}{M} f(\gamma)[x[1-(m-1) \rho \pi(d)] \\
& +(m-1) \rho \pi(d)]
\end{aligned}
$$

It is straightforward from Eq. (13) that the total throughput is an affine function on the amount of throughput $x$ to be kept by a given user. Thus, it suffices to analyze the sign of the term $(1-(m-1) \rho \pi(d))$. We have two cases:

$$
\text { Case } 1:(1-(m-1) \rho \pi(d)) \geq 0
$$

In this case $\Xi_{\text {sym }}$ reaches its maximum when $x$ is equal to 1 , i.e., all the users will satisfy their throughput demand from the base station. Here, no throughput re-injection is required to achieve the satisfaction equilibrium. Replacing $x$ by 1 in (13) yields

$$
\Xi_{\text {sym }}^{1}=\frac{m L R}{M} f(\gamma)
$$

Case 2: $(1-(m-1) \rho \pi(d)) \leq 0$

Now the total throughput $\boldsymbol{\Xi}_{\text {sym }}$ is maximized when $x$ is set to 0 . All mobile users will satisfy their whole throughput demand from the WLAN subsystem, i.e., after re-injecting their whole throughput received first from the cellular subsystem. We have the following

$$
\Xi_{\mathrm{sym}}^{0}=\frac{m(m-1) L R}{M} \rho f(\gamma) \pi(q) .
$$

Combining (14) and (15), and in order to ensure the existence of a satisfaction equilibrium, the total demand must verify:

$$
m d \leq \min \left(\Xi_{\text {sym }}^{0}, \Xi_{\text {sym }}^{1}\right),
$$

which completes the proof.

\section{Banach-Picard learning algorithm}

In this section, we seek to develop a fully distributed learning scheme to be implemented on each mobile terminal. This scheme will help to automatically learn the satisfactory solution derived above. We propose a fully distributed inspired from the well-known Banach-Picard learning algorithm [24]. Our algorithm is fully distributed in the sense that no external information is needed. Yet, a player does not need to observe the actions chosen by the other players in order to update its strategy iteratively. It only observes the realization of its own reward function that can be injected in a rule to predict its own strategy for next round.

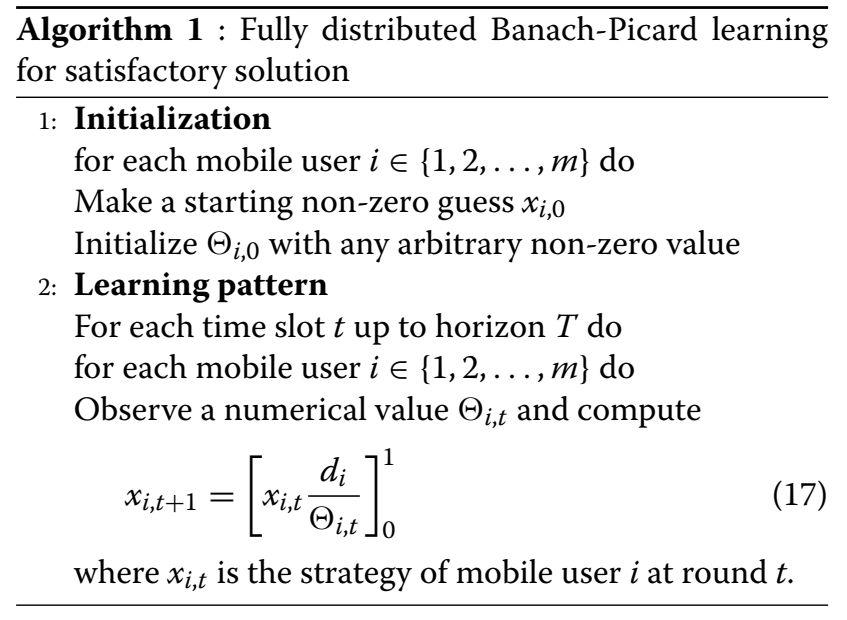

The operator $[\cdot]_{0}^{1}$ denotes the projection over the nonempty interval and continuous $[0,1]$. Denotes by $f=$ $\left(f_{i}\right)_{i \in N}$ the right-hand side of Eq. 17. Then, the set of fixed points of $f$, denoted by $f i x(f)$, belongs to the set of satisfactory solutions. The function $f$ represents the response function associated to the payoff $\Theta$. It is clear that, if the algorithm converges to some interior point $x^{*}$, then $x^{*}$ is a satisfactory solution. To prove this statement, consider a converging sequence to $x^{*}$, then the continuity of the projection map and the continuity of the payoff function implies the continuity of the quantity $x_{i, t} \frac{d_{i}}{\Theta_{i, t}}$. The stationary equation is $x_{i}^{*}=x_{i}^{*} \frac{d_{i}}{\Theta_{i}^{*}}$, i.e., the payoff of player $i$ is $\Theta_{i}^{*}=\Theta_{i}\left(x^{*}\right)=d_{i}$. This means that every player $i$ is satisfied at an interior steady state.

\section{Simulation and results}

Without loss of generality and only for sack of simplicity, we simulate here an uplink virtual MIMO system of four users. The parameters of the system are summarized in Table 1.

Regarding the efficiency function, we consider the following S-shaped function:

$$
f\left(\gamma_{i}\right)=\left(1-e^{-\gamma_{i}}\right)^{M}
$$

where $\gamma_{i}$ denotes the instantaneous signal to noise plus interference ratio given by:

$$
\gamma_{i}=\frac{p_{i} h_{i}^{2}}{\sigma_{i}^{2}+\frac{1}{N} \sum_{j \neq i} p_{j} h_{j}^{2}}
$$

$N$ is the processing gain and $h_{i}$ the channel gain of mobile user $i$. Without loss of generality and only for simplicity purpose, we consider that $h_{i}=h_{j} \quad \forall i, j$.

To examine the simulation, we propose two scenarios regarding the sharing function $\pi$ :

1. Flat-rate sharing: Here, the amount of throughput to be re-injected in the system through WLAN is 
equally shared among the other users. The explicit formula of the sharing function can write

$$
\pi_{i, j}\left(d_{1}, d_{2}, \cdots, d_{m}\right)=\frac{1}{m-1} .
$$

2. Proportional sharing: Now, the throughput to be re-injected through WLAN will be shared among other users taking into account their respective throughput demands. The expression of the sharing function in this scenario is the following:

$$
\pi_{i, j}\left(d_{1}, d_{2}, \cdots, d_{m}\right)=\frac{d_{j}}{\sum_{k \neq i} d_{k}} .
$$

In the scenarios, we examine the impact of the sharing function in VMIMO system in three cases. In first case, the users have the same quality of service and the same WLAN radio condition (same success probability). In the second case, the users have the same quality of service but different WLAN radio condition (different success probabilities). In the third case, the users have different quality of service and the same WLAN radio condition.

Case 1: The users have the same quality of service and the same probability of success:

$$
\text { - } \rho_{i}=0.5, d_{i}=70 \mathrm{Kbps} \text { for all users } i \text {. }
$$

In Fig. 3, we notice that users have the same selfproportion and the same sharing-proportion in the two scenarios. This is reasonable since that all users have the same quality of service and the same probability of success. Concerning the distribution of the sharing function, we notice that in the first scenario where the sharing proportion is divided equally between users, users prefer to share than to use the self-proportion. However, in the second scenario where the sharing proportion is divided in terms of the needs of each user, users prefer to use the self-proportion than to share.

Case 2 : The users have the same quality of service but different probability of success:
Table 2 Simulation parameters

\begin{tabular}{ll}
\hline Parameter & Value \\
\hline$p_{i}$ (power of user i) & $7 \mathrm{~W}$ \\
$\sigma^{2}$ (Gaussian noise) & $25 \times 10^{-16} \mathrm{~W}$ \\
$\mathrm{R}$ & $100 \mathrm{Kbps}$ \\
$\mathrm{M}$ & 100 \\
$\mathrm{~L}$ & 100 \\
$\mathrm{~d} 1$ & $60 \mathrm{Kbps}$ \\
$\mathrm{d} 2$ & $20 \mathrm{Kbps}$ \\
$\mathrm{d} 3$ & $40 \mathrm{Kbps}$ \\
$\mathrm{d} 4$ & $75 \mathrm{Kbps}$ \\
$\rho_{1}$ & 0.2 \\
$\rho_{2}$ & 0.2 \\
$\rho_{3}$ & 0.2 \\
$\rho_{4}$ & 0.2 \\
\hline
\end{tabular}

$-\rho_{1}=0.1, \rho_{2}=0.3, \rho_{3}=0.6, \rho_{4}=0.9$.

$-d_{i}=70 \mathrm{Kbps}$ for all users $i$.

In Figs. 4 and 5, we notice that the probability of success has an impact on the distribution of the proportion even if users have the same quality of service. This is justified by the fact that the user who has a high probability of success will tend to use the self-proportion more than to use the sharing-proportion and vice versa. In the first scenario where the sharing proportion is divided equally between users, users prefer to share than to use the self-proportion. In the second scenario where the sharing proportion is shared on the basis of the need of each user, we notice that users prefer to use the self-proportion than sharing proportion.

Case 3 : The users have different quality of service and the same probability of success:

$$
\begin{aligned}
& -\rho_{i}=0.5 \text { for all users } \mathrm{i} . \\
& -d_{1}=40 \mathrm{Kbps}, d_{2}=80 \mathrm{kbps}, d_{3}=60 \mathrm{Kbps}, \\
& d_{4}=100 \mathrm{kbps}
\end{aligned}
$$

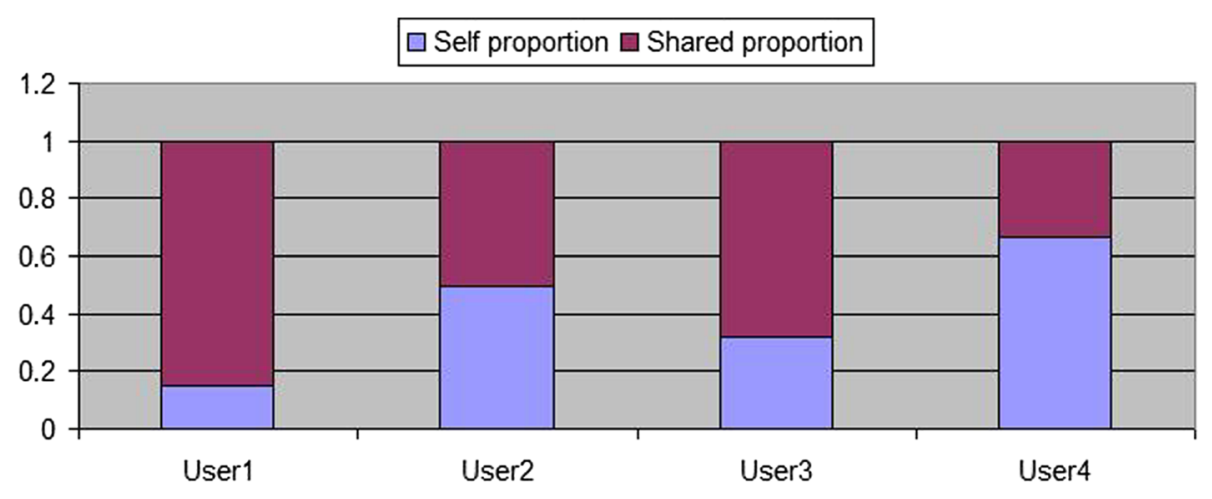

Fig. 6 Distribution of self and shared uplink proportion for four users in first scenario 


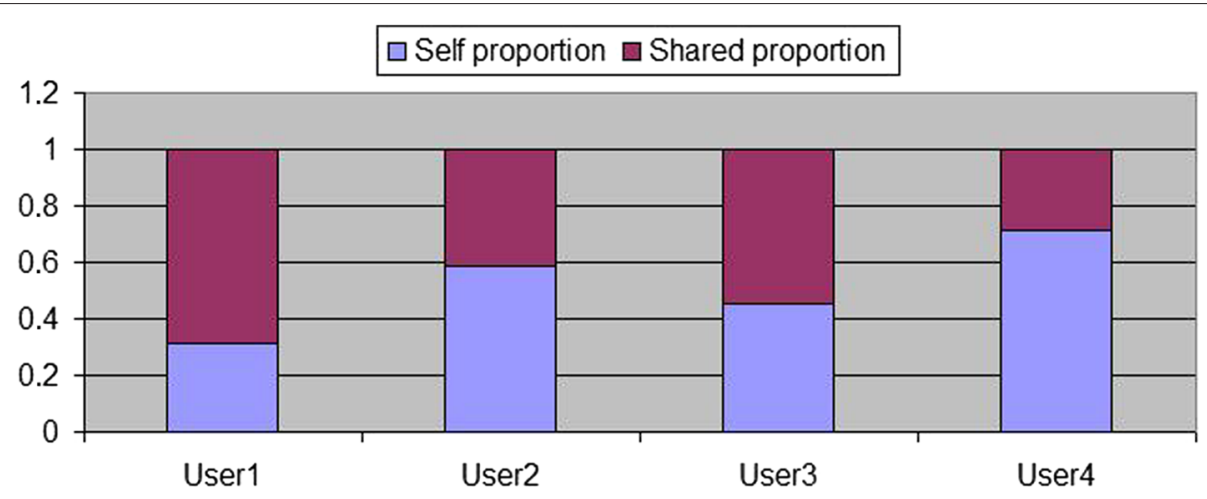

Fig. 7 Distribution of self and shared uplink proportion for four users in second scenario

In Figs. 6 and 7 we notice that the quality of service has great impact on the distribution of the proportion even if users have the same probability of success. This is justified by the fact that the user who needs a high quality of service will not share much and vice versa. In the first scenario where the sharing proportion is divided equally between users, users prefer to share than to use the self-proportion. In the second scenario where the sharing proportion is shared on the basis of the need of each user, we notice that users prefer to use the self-proportion than sharing proportion.

The fixed parameters of the simulation are shown in Table 2.

\subsection{Results and analysis}

As depicted in Fig. 8, the evolution of the cellular proportion of all users and convergence to stable proportion when they meet Nash equilibrium after less than 10 iterations.

Figure 9 shows the evolution of the demanded throughput (utility) per user.
The condition of Nash equilibrium has been meeting in iteration which is nearly 5 , and each user has no intention to change his strategies to improve his throughput. This result observed after the iteration 5 is the best that each user can achieve.

\section{Conclusions}

In this work, we studied a constrained non-cooperative game analysis to achieve QoS in VMIMO systems using a approach. A non-cooperative game is proposed in order to study the interaction among users, where each user (selfishly) decides the appropriate amount of throughput to re-inject in the WLAN network in order to satisfy its throughput requirement. Yet, the equilibrium is achieved when demanded QoS is met for all users. We showed that there is a unique satisfaction equilibrium for the proposed game; we characterized it and computed it explicitly for general case. Next, we proposed a fully distributed algorithm to learn the satisfaction equilibrium. Then, using the proposed scheme over an uplink virtual MIMO system with $m$ users, we discussed the effect of asked QoS and

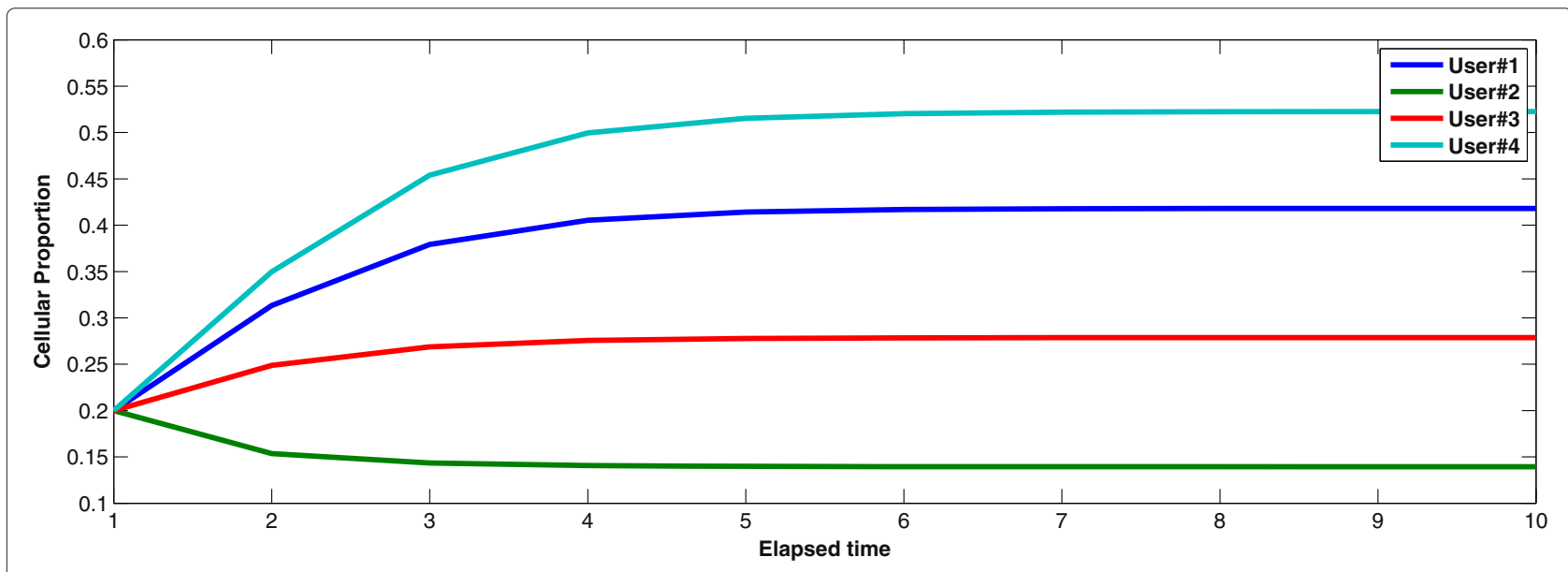

Fig. 8 Evolution of the cellular proportion per user 


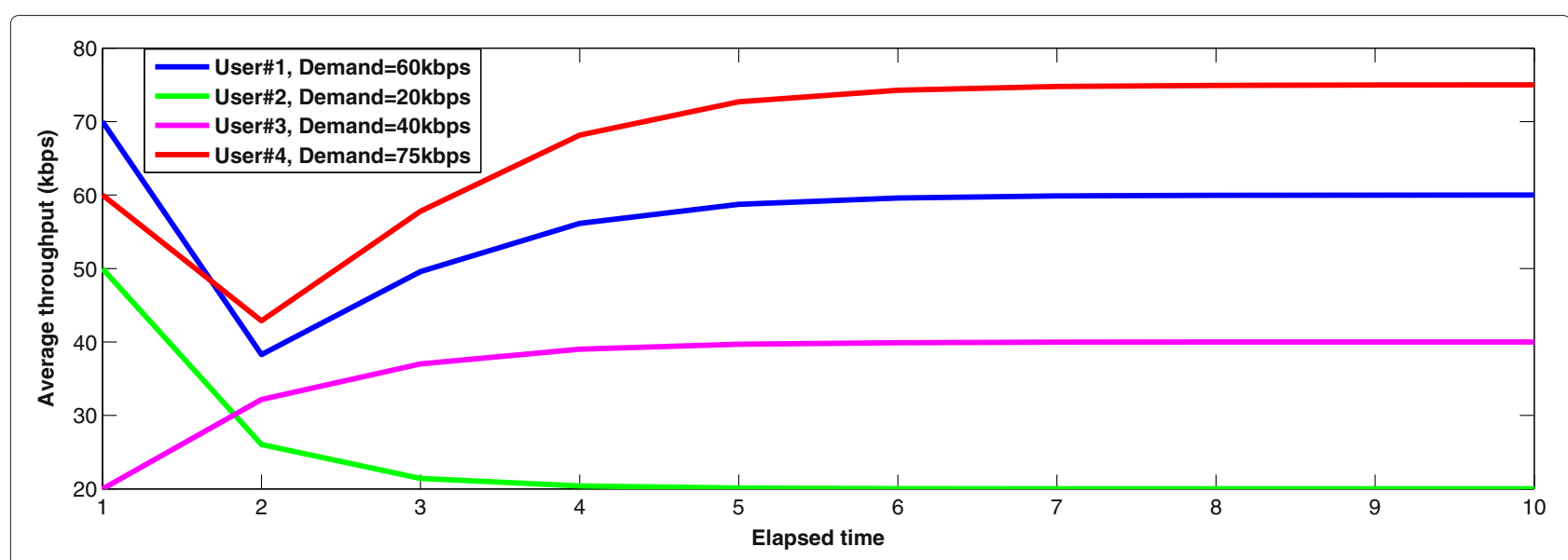

Fig. 9 Evolution of the demand throughput per user

the WLAN radio condition that have a substantial impact on the amount of throughput to be re-injected in the system.

Next, our analytical results are corroborated with extensive simulation results. We considered many schemes regarding the sharing function. This latter function shows how re-injected throughput must be shared among users inside WLAN range of injecting user. In particular, we analyzed the case of flat-rate sharing and the case of proportional sharing. We found that the results achieved in the proportional sharing scenario are more interesting compared to those achieved in the flat-rate scenario. Moreover, proportional sharing scheme presents better fairness properties; this is because the distribution of the sharing-rate depends on the demand of each user. Finally, simulations show that our Banach-Picard-inspired algorithm converges well and super fast to the satisfaction equilibrium which is very appreciated in this kind of communications.

As a future direction, we are working towards adapting our model to support dense and ultra dense networks such as Massive MIMO and ultra dense device-to-device communications using evolutionary game theory and mean field dynamic game theory.

\section{Endnotes}

${ }^{1}$ Nowadays, smart devices are usually equipped with several interfaces, e.g., 2G, 3G, 4G, WLAN, Bluetooth, NFS, and Ir).

${ }^{2}$ Note that power allocation problem is outside of the scope of this paper. However, fortunately and since our proposed learning algorithm presented in Section 5 is very fast, one could efficiently/dynamically combine the VMIMO game and the poser control game.

\section{Competing interests}

The authors declare that they have no competing interests.

\section{Acknowledgements}

We would like to thank so much the anonymous referees for their effort, their time, and their valuable inputs that invariably improve this article. We also would like to thank Professor Tembiné Hamidou (from New York University) for his very constructive comments and suggestions.

\section{Author details}

${ }^{1}$ ENSIAS, Mohammed V University, BP 713, 10000 Rabat, Morocco. ${ }^{2}$ NEST Research Group, ENSEM, Hassan II University, Route d'El Jadida BP 8118 Oasis, 20000 Casablanca, Morocco. ${ }^{3}$ University of Paris 13, 99 Avenue Jean-Baptiste Clement, 93430 Villetaneuse, France.

Received: 12 March 2015 Accepted: 13 April 2016

Published online: 06 May 2016

\section{References}

1. $\mathrm{H} \mathrm{Ji}, \mathrm{C}-\mathrm{Y}$ Huang, Non-cooperative uplink power control in cellular radio systems. Wireless Netw. 4, 233-240 (1998)

2. DJ Goodman, NB Mandayam, in Vehicular Technology Conference, 2001. VTC 2001 Spring. IEEE VTS 53rd. Network Assisted Power Control for Wireless Data, vol. 2, (2001), pp. 1022-1026. doi:10.1109/NETECS.2001.944533 ISSN:1090-3038

3. CU Saraydar, NB Mandayam, DJ Goodman, Pricing and power control in a multicell wireless data network. IEEE J. Selected Areas Commun. 19, 1883-1892 (2001)

4. CU Saraydar, NB Mandayam, DJ Goodman, Efficient power control via pricing in wireless data networks. IEEE Trans. Commun. 50, 291-303 (2002)

5. T Alpcan, T Basar, R Srikant, E Altman, in Decision and Control, 2001. Proceedings of the 40th IEEE Conference on. CDMA Uplink Power Control as a Noncooperative Game, vol. 1, (2001), pp. 197-202. doi:10.1109/.2001.980097

6. CW Sung, WS Wong, A noncooperative power control game for multirate CDMA data networks. IEEE Trans. Wireless Commun. 2, 186-194 (2003)

7. N Feng, S-C Mau, NB Mandayam, Pricing and power control for joint network-centric and user-centric radio resource management. IEEE Trans. Commun. 52, 1547-1557 (2004)

8. F Meshkati, M Chiang, SC Schwartz, HV Poor, NB Mandayam, in IEEE Wireless Communications and Networking Conference, 2005. A Non-cooperative Power Control Game for Multi-carrier CDMA Systems, vol. 1, (New Orleans, LA, USA, 2005), pp. 606-611. doi:10.1109/WCNC.2005.1424570 ISSN:1525-3511

9. SCui, AJ Goldsmith, A Bahai, Energy-efficiency of MIMO and cooperative MIMO techniques in sensor networks. IEEE J. Select. Areas. Commun. 22(6), 1089-1098 (2003)

10. SK Jayaweera, Virtual MIMO-based cooperative communication for energy-constrained wireless sensor networks. IEEE Trans. Wireless Commun. 5(5), 984-989 (2008) 
11. Y Yuan, ZH He, M Chen, Virtual MIMO-based cross-layer design for wireless sensor networks. IEEE Trans. Vehicular Technol. 55(3), 856-864 (2009)

12. W Cheng, X Zhang, H Zhang, QoS-aware power allocations for maximizing effective capacity over virtual-MIMO wireless networks. Selected Areas Commun. IEEE J. 31, 2043-2057 (2013)

13. X Hongli, H Liusheng, Q Chunming, W Xinglong, S Yu-e, Topology control with VMIMO communication in wireless sensor networks. Wireless Commun. IEEE Trans. 12, 6328-6339 (2013)

14. H Bennani, E Sabir, A Kobbane, A Walid, J Ben-Othman, in 2014 International Wireless Communications and Mobile Computing Conference (IWCMC). A Tax-Inspired Mechanism Design to Achieve QoS in VMIMO systems: Give to Receive, (Nicosia, Cyprus, 2014), pp. 56-62. doi:10.1109/IWCMC.2014.6906332 ISSN:2376-6492

15. H Chen, Z Pan, L Tian, J Shi, G Yang, M Suzuki, A novel AWSF algorithm for doa estimation in virtual MIMO systems. Selected Areas Commun. IEEE J. 31, 1994-2003 (2013)

16. OB Karimi, MA Toutounchian, J Liu, C Wang, Lightweight user grouping with flexible degrees of freedom in virtual MIMO, selected areas in communications. IEEE J. 31, 2004-2012 (2013)

17. S Park, JW Choi, JY Seol, B Shim, in Information Theory and Applications Workshop (ITA), 2014. Virtual pilot signal for massive MIMO-OFDM systems, (2014). doi:10.1109/ITA.2014.6804285

18. HXu, L Huang, C Qiao, W Dai, S Yu-e, Joint virtual MIMO and data gathering for wireless sensor networks. Parallel Distributed Syst. IEEE Trans. 99, 1 (2014)

19. W Lu, Y Liu, D Wang, Efficient feedback scheme based on compressed sensing in MIMO wireless networks. J. Comput. Electr. Eng. 39, 1587-1600 (2013)

20. A Thampi, S Armour, Z Fan, D Kaleshi, in European Wireless 2014; 20th European Wireless Conference; Proceedings of. Clustered Network MIMO and Fractional Frequency Reuse for the Downlink in LTE-A Systems, (2014), pp. 1-6

21. Y Xu, J Wang, Q Wu, A Anpalagan, YD Yao, Opportunistic spectrum access in unknown dynamic environment: A game theoretic stochastic learning solution. IEEE Trans. Wireless Commun. 11(4), 1380-1391 (2012)

22. $Y X u$, J Wang, Q Wu, A Anpalagan, YD Yao, Opportunistic spectrum access in cognitive radio networks: Global optimization using local interaction games. IEEE J. Selected Topics Signal Process. 6(2), 180-194 (2012)

23. E Sabir, R El-Azouzi, Y Hayel, Hierarchy sustains partial cooperation and induces a Braess-like paradox in slotted aloha-based networks. Comput. Commun. 35(3), 273-286 (2012)

24. HTembine, R Tempone, P Vilanova, in 52nd IEEE Conference on Decision and Control. Mean-Field Learning for Satisfactory Solutions, (2013), pp. 4871-4876. doi:10.1109/CDC.2013.6760653 ISSN:0191-2216

\section{Submit your manuscript to a SpringerOpen ${ }^{\circ}$ journal and benefit from:}

- Convenient online submission

- Rigorous peer review

- Immediate publication on acceptance

- Open access: articles freely available online

- High visibility within the field

- Retaining the copyright to your article

Submit your next manuscript at $\boldsymbol{\nabla}$ springeropen.com 